\section{FORMACIÓN CONTINUA EN CENTROS DE FP Y ACTIVIDADES DE INNOVACIÓN EN LAS PYMES INDUSTRIALES}

\author{
Cristina Lavía \\ Mikel Olazaran \\ Eneka Albizu \\ Beatriz Otero
}

Universidad del País Vasco/Euskal Herriko Unibertsitatea

(UPV-EHU)

\begin{abstract}
The current economic crisis is affecting Spain with special force. Within that context, the relationships between the education system and the economic system become a key issue. The aim of this paper is to make a contribution to the understanding of the relationships between vocational training (VT) centres and small and medium-sized firms (SMEs). Our study is based on a survey of ind ustrial SMEs from two Northern Spanish autonomous communities (Basque Country and Navarre). The different types of SME-VT centre relationship are analyzed, and special attention if devoted to new forms of relationships among those agents (mainly continuous training), and to the possible effects of SME-VT centre collaboration upon the firms' innovation processes. We show that, right now, continuous training activities are a key form of relationship between VT centres and SMEs. That type of relationship acquires an strategic importance from the point of view of the coordination between the training system and the innovation system.
\end{abstract}

KEY WORDS: Regional innovation systems; vocational training; small and medium-sized firms (SMEs); economic sociology; Spain.

\section{INTRODUCCIÓN}

En el contexto actual de crisis económica, con sus dramáticos efectos sociales que se manifiestan en indicadores como el nivel de desempleo, es urgente avanzar en el conocimiento y reflexión sobre las relaciones entre el sistema educativo y el sistema económico. Un aspecto destacable en España es el bajo porcentaje de personas con formación secundaria postobligatoria (bachillerato y formación profesional de grado medio): $22 \%$ de la población de 25 a 64 años en 2007, 21 puntos por debajo de la media de la OCDE (OCDE, 2009). El porcentaje de población con este nivel de estudios ha aumentado en 9 puntos en los últimos años. Para el conjunto de la OCDE ha permanecido

\section{CONTINUOUS TRAINING, VOCATIONAL EDUCATION CENTRES AND INNOVATION ACTIVITIES IN INDUSTRIAL SMES}

RESUMEN: La crisis actual, que afecta con especial virulencia a España, obliga a reflexionar sobre las relaciones entre el sistema educativo y el sistema económico. En este trabajo, basado en una encuesta a pequeñas y medianas empresas industriales de las comunidades autónomas vasca y navarra, se pretende avanzar en el conocimiento de las relaciones entre los centros de Formación Profesional y las empresas. Se prestará especial atención a las nuevas formas de relación entre ambos agentes (especialmente la formación continua), y al efecto que la colaboración con los centros de FP pueda tener sobre los procesos de innovación de las empresas. Se muestra que, en la actualidad, las actividades de formación continua constituyen una forma de colaboración importante para las empresas y estratégica desde el punto de vista del ajuste entre el sistema de formación y el sistema de innovación.

PALABRAS CLAVE: Sistemas regionales de innovación; formación profesional; pequeñas y medianas empresas (pymes); sociología económica; España.

constante en torno al $43 \%$, por lo que la distancia sigue siendo muy importante².

Aún estando ampliamente reconocido el valor de la Formación Profesional en el desarrollo económico y social (Cedefop, 2009), el Estado español presenta unos índices bajos si se comparan con los principales países europeos. En el curso académico 2007/2008, la tasa bruta de escolarización ${ }^{3}$ en FP de Grado Medio, uno de los talones de Aquiles del sistema educativo, era del 26,6\%, mientras que la de Grado Superior, alcanzaba el 23,7\%. En la mayoría de los países desarrollados o en vías de desarrollo, estos estudios tienen mayor repercusión social e impacto en la economía. En Alemania y en Austria, por ejemplo, 
la mayoria de los alumnos de la Enseñanza Secundaria siguen la rama profesional. En países sumidos en procesos de desarrollo socio-económico como China, India, Rusia o Korea del Sur, cerca del $40 \%$ de las personas en edad de cursar estudios secundarios opta por la FP. La media de la UE (sin Reino Unido) para estudios secundarios de FP en 2006 ha sido el 49\%.

En los últimos años se han llevado a cabo importantes reformas en el sistema de Formación Profesional, como son, la implantación de los nuevos grados Medio y Superior a partir de la LOGSE, la institucionalización de los convenios con las empresas para la Formación en Centros de Trabajo (FCT) y la revisión de los planes de estudios en base a competencias y módulos profesionales. Siguen su curso en la actualidad los cambios dirigidos al desarrollo del Sistema Nacional de Cualificaciones y la integración de los distintos sistemas de formación (formación para el empleo y formación profesional). Se han dado pasos significativos, pero quedan retos muy importantes por abordar, tanto en el sistema educativo (destaca el fracaso y abandono escolar) como en las relaciones entre éste y el sistema económico (los objetivos de ambos sistemas han sido escasamente convergentes, en general).

En este trabajo, basado en una encuesta a pymes industriales de las comunidades autónomas vasca y navarra, queremos avanzar en el conocimiento de las relaciones entre los centros de FP y las empresas, con especial atención a las nuevas funciones que comienzan a desarrollar los centros (sobre todo, las actividades de formación continua) y a la incidencia que ello pudiera tener en los procesos de innovación de las empresas.

Se trata de un trabajo exploratorio, diseñado a partir de una primera fase de investigación cualitativa (basada en 11 entrevistas a directores de centros de FP) y del análisis de los principales datos de la encuesta, que pretende responder a las siguientes preguntas de investigación:

- ¿Cómo se relacionan los centros de FP y las empresas y cuál es el alcance de los distintos tipos de relación?

- ¿Qué valoración realizan las empresas de los distintos tipos de relación?

- ¿Qué importancia tiene la formación continua como una misión "nueva", o "no tradicional" de los centros de FP?
- ¿Existe una relación entre el carácter innovador de una empresa y sus pautas de relación con los centros de FP?

- Y, por último: ¿cómo se valora el papel que juegan, o pueden jugar, en los procesos de innovación de las empresas, los trabajadores con perfil de FP?

La estructura del documento es la siguiente. Después de esta introducción, en el apartado segundo se explicita el marco conceptual dentro del cual se ha realizado la investigación. Se parte de la perspectiva del sistema de innovación, y se revisa el papel que la formación profesional y los trabajadores de FP pueden tener en los procesos de innovación. En el apartado tercero se da cuenta del diseño de investigación y de la metodología empleada. En los siguientes apartados se presentan los resultados obtenidos relativos a las siguientes dimensiones:

- Actividades de innovación y formación en las empresas (apartado cuarto).

- Relaciones entre los empresas y los centros de FP, con especial atención a la formación continua (apartado quinto).

- Valoración del papel de los trabajadores de FP en los procesos de innovación de las empresas (apartado sexto).

Finalmente, en el apartado séptimo se exponen las principales conclusiones de la investigación.

\section{Sistema de INNOVACIÓN Y FORMACIÓN PROFESIONAL}

Nuestro marco conceptual se sitúa dentro de la perspectiva de los sistemas de innovación, y más en concreto, en el "modelo interactivo" de innovación estudiado por Lundvall y colaboradores en el caso danés. La perspectiva del sistema nacional de innovación surgió con fuerza en los años 90, poniendo en cuestión los postulados ortodoxos sobre el papel del conocimiento como genérico, codificable, accesible sin coste e independiente del contexto (Freeman, 1987; Dosi et al.; 1988, Lundvall, 1992a; Nelson, 1993; Edquist, 1997). Esta perspectiva estudia la innovación como fenómeno sistémico, donde se interrelacionan actores y factores tanto internos como externos a la empresa, dentro 
de un entorno institucional y cultural donde las relaciones entre el sector público y el privado, el sistema educativo y productivo, el marco de relaciones laborales y el sistema financiero tienen una gran importancia. El cambio técnico sería un proceso endógeno, dependiente de una trayectoria y condicionado por un contexto organizacional, institucional y cultural.

Con su noción de "interactive learning", Lundvall y equipo han profundizado en el carácter interactivo de los procesos de innovación, que son el resultado, por un lado, de la interacción continua entre agentes internos a la empresa (por ejemplo entre diseño, producción y marketing, entre ingenieros y trabajadores de producción, o entre personal de la Oficina Técnica y Servicio de Asistencia Técnica) y, por otro, de las relaciones entre la organización y agentes externos (principalmente, clientes y proveedores). A los distintos tipos de aprendizaje estudiados con anterioridad por la teoría evolutiva, como "learning by doing" (aprender haciendo, en las actividades productivas habituales, resolviendo problemas) y "learning by using" (aprender en base al uso y adaptación de nuevos sistemas técnicos), Lundvall añade el "learning by interacting", tanto interno a la organización (en base al trabajo en equipos multidisciplinares), como externo a la misma, notablemente, en las relaciones productor-usuario, típicas, por ejemplo, de la relación entre fabricantes especializados y grandes clientes.

La aplicación del concepto de sistema de innovación a nivel regional ha ganado una creciente importancia política como instrumento para analizar los procesos de innovación a dicha escala. Con el concepto de sistema regional se subraya la importancia de la escala regional y de los recursos específicos regionales en la innovación de las empresas -especialmente pymes- y en la competitividad de las regiones (Braczyk et al., 1996; Storper, 1997; Cooke y Morgan, 1998; Maskell y Malmberg, 1999).

Las características institucionales de una región y sus infraestructuras de conocimiento se consideran importantes condiciones básicas que pueden servir de estímulo para promover las actividades innovadoras (Isaksen y Asheim, 2003). Diferentes estudios destacan que la región es especialmente importante para el intercambio de conocimiento tácito y para la provisión de mano de obra cualificada, ambos mecanismos muy importantes en los procesos de innovación de las empresas pequeñas (Asheim y Coenen,
2005; Kauffmann y Tödtling, 2003; Grotz y Braun, 1997; Doloreux 2003; Gebauer et al., 2005).

No obstante, a nuestro entender, desde la perspectiva del sistema de innovación no se ha prestado atención suficiente a la relación entre el sistema de formación y el sistema de innovación. Como han indicado recientemente algunos autores, el sistema de innovación está "enraizado" en el sistema de producción y en el sistema de "desarrollo de los recursos humanos" (Lundvall, 2002; Lundvall y Christensen, 2003). Los sistemas de educación y el mercado laboral juegan un papel crucial en la construcción de competencias y capacidades de innovación.

En los últimos años, algunos estudios trasnacionales han centrado su atención en el análisis de las diferencias institucionales, el aprendizaje intra-organizacional y el estilo de innovación (Arundel et al., 2007; Lundvall et al., 2008). En estos trabajos se señala que lo que impide mejorar la capacidad innovadora de las empresas en algunos paises europeos puede que no sea tanto el bajo nivel de inversión en $I+D$ sino, más bien, la existencia de estructuras organizacionales que obstaculizan la innovación (Arundel et al., ibíd., p. 1204). Entre las caracteristicas de estas estructuras se menciona el débil papel de la formación profesional y de los trabajadores que tienen este perfil.

"One bottleneck for constructing learning organisations in the less-developed economies of Europe would appear to be at the level of vocational training. Portugal, Spain, Italy and Greece, all of which have made important strides in increasing the level of science and engineering graduates, stand out for their low levels of investment in continuing vocational training and for ranking lowest on the discretionary learning scale" (Lundvall et al., ibíd., p. 689).

Recientemente, algunos autores han destacado la importancia que tiene el sistema de formación profesional y continua en la innovación (Rosenfeld, 1998; Curtain, 2004; Guthrie y Dawe, 2004; Toner, Marceau, Hall y Considine, 2004; Whittingham, Ferrier y Trood, 2004; Tether, Mina, Consoli y Gagliardi, 2005; Moodie, 2006; Bosch \& Charest, 2008; Ranga, Miedema y Jorna, 2008; Toner, 2009). En un importante informe que Stuart Rosenfeld realizó para la Organización para la Cooperación y Desarrollo Económico (OCDE), el autor sugirió que los institutos de educación y formación profesional técnica estaban en mejor posición

ARBOR Vol. 188753 enero-febrero [2012] 153-170 ISSN: 0210-1963

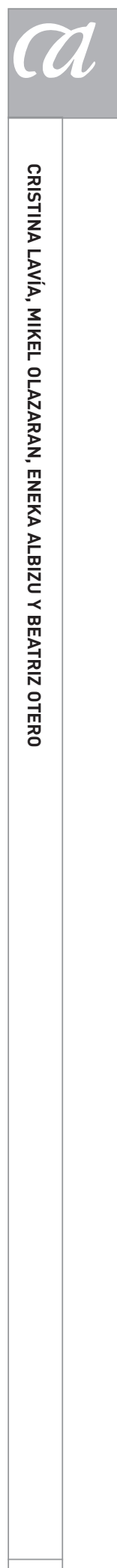


que las universidades para dar servicio a las pymes, "en una era en la que los empleadores aseguran que el verdadero problema es la falta de técnicos, y no de ingenieros" (Rosenfeld, ibíd., pp. 6 y 35).

Rosenfeld propone una interesante taxonomía de las misiones de los institutos de Formación Profesional (FP), que abarcan la educación reglada, la formación continua y el apoyo a las pymes en la implementación de nuevas tecnologías, en los procesos de innovación y en la cooperación con otras empresas. Éste y otros estudios destacan la importancia de que el personal de los institutos de FP tenga experiencia en la industria, asi como el papel que pueden jugar dichos institutos en los sistemas de innovación locales y regionales. Sin embargo, también se apunta que la participación de los centros de FP en misiones diferentes a las tradicionales es obstaculizada a menudo por diferentes barreras, como son la falta de autonomía o flexibilidad de los institutos, la falta de incentivos o de instrumentos públicos de fomento y la falta de relación con agentes de $I+D$, tales como los centros tecnológicos (Guthrie et al., ibíd., p. 14; Whittingham et al., ibíd., p. 115).

Como señalan Toner y Tether, entre otros, se tiende a olvidar que los trabajadores técnicos con cualificaciones intermedias intervienen en el diseño, funcionamiento y mantenimiento de los productos y procesos, y por tanto contribuyen (o pueden contribuir) a la innovación en las empresas. Las empresas innovadoras necesitan una plantilla cualificada e implicada en la mejora continua de procesos y productos (innovación incremental, "aprender haciendo").

En suma, la complementariedad entre educación, formación e innovación parece clara. No obstante, tal y como indica Phillip Toner (2009, p. 61, traducción nuestra), "aunque se reconoce el papel central para el fomento de la innovación de una estructura de cualificaciones técnicas intermedias más distribuidas y de más nivel conceptual, se han hecho pocos trabajos en el campo de los estudios de la innovación, o de otras disciplinas, en lo que respecta a este tema".

En España el proceso de reforma del sistema de $\mathrm{FP}$, que comenzó en los 90 y que en los últimos años se ha acrecentado, no ha conseguido todavía neutralizar problemas como el bajo nivel de cualificaciones secundarias post- obligatorias y no universitarias (OCDE, 2009), la insuficiente relación entre la formación profesional y las necesidades de la industria (CES, 2009, p. 250), la falta de un sistema de cualificaciones y de reconocimiento de competencias, la falta de coordinación entre el sistema de educación profesional y el sistema de formación para el empleo (Homs, 2008; CES, ibíd.) y los problemas de gobernanza entre los niveles estatal y regional (Homs, ibíd.). Por otro lado, el problema de los costes y beneficios de la formación parece estar muy presente, existiendo un temor por parte de los empresarios a que unos trabajadores más formados pudieran dejar la empresa o demandar subidas salariales (Berechet et al. 2008, p. 69). Finalmente, el modelo organizativo jerárquico dominante en buena parte del tejido empresarial, caracterizado por un nivel bajo de cualificaciones y tareas rutinarias, no parece favorecer la formación y la innovación (Huerta, 2003).

\section{Metodología}

En este trabajo se pretende aumentar el conocimiento sobre las relaciones entre los centros de FP y las empresas, con especial atención a las nuevas misiones de los centros como son la formación continua y el apoyo a la innovación. Se parte de una primera fase de investigación cualitativa realizada en el segundo semestre de 2009 en la cual, en base a entrevistas a once directores de centros de FP, se identificaron los principales tipos de relaciones entre los centros y las empresas, así como las potencialidades y problemas de cada uno de ellos (principalmente, prácticas FCT, formación continua y apoyo a la innovación).

A partir de ahí se construyó un cuestionario al objeto de recoger la valoración de las pequeñas y medianas empresas industriales (principales destinatarias del capital humano y los servicios de los centros) sobre los distintos tipos de relación y responder a las preguntas de investigación recogidas en la introducción de este documento.

Se toma como unidad de análisis el conjunto de las comunidades autónomas vasca y navarra, por considerar que en dichos territorios la FP presenta un grado de desarrollo y relación con la empresa industrial importante y bastante homogéneo. Estas comunidades comparten algunas características similares, como la importancia del sector indus- 
trial y de las políticas regionales. Al considerar el conjunto de dichas CCAA hemos pretendido también aumentar el alcance del estudio cuantitativo.

El estudio se ha realizado considerando como población a todas las empresas de entre 25 y 249 empleados/as (pymes) con domicilio social en las CCAA del País Vasco y Navarra4.

Se ha seleccionado una muestra aleatoria $(N=329)$ de entre la población de las 1.718 empresas de entre 25 y 249 trabajadores (pymes) que se pueden considerar industriales/manufactureras (códigos CNAE93 entre 15 y 41). El tamaño de muestra global (329 empresas) garantiza la representatividad estadística del colectivo de empresas con un error muestral máximo del 5\% para estimaciones a un NC95, 5\% en el supuesto de diversidad más desfavorable $(p=q=0,5)$. La muestra fue estratificada de modo proporcional por territorios (provincias) y tres niveles de tamaño, con selección final aleatoria dentro de cada estrato.

La obtención de la información pretendida a partir de la muestra seleccionada se ha realizado a través de entrevistas telefónicas. El cuestionario que se ha utilizado para la realización del trabajo de campo ha sido elaborado por el propio equipo de investigación, tomando como referencia: los objetivos de la investigación, la literatura analizada, los resultados de la investigación cualitativa efectuada en la primera fase del proyecto y las limitaciones inherentes a la modalidad de encuesta seleccionada (telefónica).

La encuesta se realizó a los responsables de las pymes en función, principalmente, de su especialización y en orden jerárquico ${ }^{5}$. Así, en el $85 \%$ de las empresas se entrevistó a responsables de recursos humanos y formación, y en el resto, a gerentes o directores.
Con respecto a los sectores de actividad de las empresas, la muestra ha resultado considerablemente variada, destacando especialmente algunos tipos como: Fabricación de productos metálicos (30,1\% de empresas), Industrias de la construcción de maquinaria y equipo mecánico $(18,8 \%)$, Industrias de productos alimenticios/bebidas (7,6\%), Fabricación de productos del caucho y materias plásticas (7\%) o Metalurgia $(4,9 \%)$. En total, más de la mitad $(63,8 \%)$ de las empresas pueden clasificarse en la agrupación del tipo Metalmecánica ${ }^{6}$ frente al resto, $36,2 \%$ de actividades más diversas.

No se puede hablar de una relación relevante entre sector y tamaño de las empresas, si bien, entre las empresas de metalmecánica destacan algo más las de mayor tamaño frente a una distribución de tamaños algo más heterogénea para las empresas dedicadas al resto de actividades.

Es importante señalar que la distribución por sectores es desigual según provincias: el sector metalmecánico está especialmente presente en la estructura de actividad de las pymes guipuzcoanas y también vizcaínas, que son, por tamaño, las que absorben más cantidad de muestra, con lo que contribuyen más a las caracteristicas de la distribución de sectores del conjunto.

A continuación se presentan los resultados descriptivos obtenidos de la encuesta realizada, organizados en torno a tres apartados:

- Actividades de innovación y formación en las pymes industriales.

- Relaciones que se establecen entre los centros de FP y las pymes industriales, con especial atención a la formación continua.

TABLA 1. DISTRIBUCIÓN DE LA MUESTRA POR TAMAÑOS

\begin{tabular}{|l|c|c|c|c|c|c|c|c|}
\hline & $\mathbf{2 5 - 7 4}$ & $\%$ & $\mathbf{7 5 - 1 4 9}$ & $\%$ & $\mathbf{1 5 0 - 2 4 9}$ & $\%$ & TOTAL & $\%$ \\
\hline Álava & 37 & 71,2 & 10 & 19,2 & 5 & 9,6 & 52 & 15,8 \\
\hline Guipúzcoa & 71 & 75,5 & 17 & 18,1 & 6 & 6,4 & 94 & 28,6 \\
\hline Vizcaya & 75 & 73,5 & 19 & 18,6 & 8 & 7,8 & 102 & 31,0 \\
\hline Navarra & 61 & 75,3 & 14 & 17,3 & 6 & 7,4 & 81 & 24,6 \\
\hline Total & 244 & 74,2 & 60 & 18,2 & 25 & 7,6 & 329 & 100,0 \\
\hline
\end{tabular}


- Importancia de los trabajadores de FP en los procesos de innovación.

En cada uno de estos apartados se analizarán los resultados generales de la encuesta, a la vez que se tendrá en cuenta si existen diferencias según variables como el tamaño empresarial, el sector de actividad, las actividades de innovación y/o la existencia de planes de formación.

\section{ACtiVIDADES DE INNOVACIÓN Y FORMACIÓN EN LAS PYMES INDUSTRIALES}

\subsection{Actividades de innovación}

El 66\% de las empresas encuestadas se declaran innovadoras. Dentro de esta tendencia mayoritaria, se observa que las empresas de tamaño más grande presentan una tasa de innovación superior que las pymes más pequeñas
(74,2 frente a $60,2 \%)$. Esta relación es estadisticamente significativa $(\chi 2$ sig. $=0,009)$.

Las innovaciones que realizan las empresas son principalmente de producto (53\% de las que dicen innovar) $y$, secundariamente, de proceso (26\%). Muy por detrás se encuentran las innovaciones organizativas $(11 \%)$ y de mercados (9\%).

Entre las empresas de más de 50 empleados se da una mayor presencia de las innovaciones de producto. Cabe mencionar también la mayor importancia de la innovación organizativa en las empresas pequeñas.

En cuanto a los sectores (metalmecánico vs resto), el nivel de innovación en producto (ligeramente), y de forma más marcada, en organización y mercados, es superior en el sector metalmecánica, mientras que la innovación en procesos es más frecuente (13,2 puntos porcentuales) en las empresas pertenecientes al resto de los sectores.

TABLA 2. EMPRESAS INNOVADORAS SEGÚN TAMAÑO Y SECTOR

\begin{tabular}{|c|c|c|c|c|c|c|c|}
\hline & & \multicolumn{6}{|c|}{ ¿Considera que su empresa es innovadora? } \\
\hline & & \multicolumn{2}{|c|}{ sí } & \multicolumn{2}{|c|}{ NO } & \multicolumn{2}{|c|}{ Total } \\
\hline & & $\mathrm{N}$ & $\%$ & $\mathrm{~N}$ & $\%$ & $\mathrm{~N}$ & $\%$ \\
\hline \multirow{2}{*}{ TAMAÑO } & HASTA 50 & 121 & 60,2 & 80 & 39,8 & 201 & 100,0 \\
\hline & MÁS DE 50 & 95 & 74,2 & 33 & 25,8 & 128 & 100,0 \\
\hline \multirow{2}{*}{ SECTOR } & Empresas de metalmecánica & 138 & 65,7 & 72 & 34,3 & 210 & 100,0 \\
\hline & Resto de empresas & 78 & 65,5 & 41 & 34,5 & 119 & 100,0 \\
\hline \multicolumn{2}{|l|}{ TOTAL } & 216 & 65,7 & 113 & 34,3 & 329 & 100,0 \\
\hline
\end{tabular}

TABLA 3. TIPOS DE INNOVACIÓN SEGÚN TAMAÑO Y SECTOR DE LAS EMPRESAS

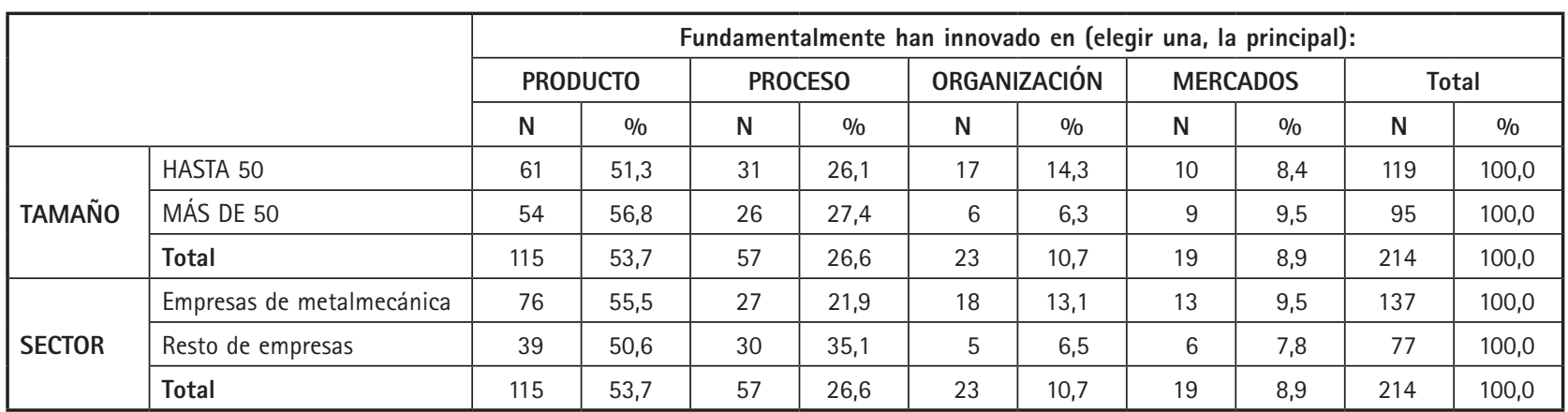




\subsection{Actividades de formación}

La inmensa mayoria de las empresas dice contar con algún tipo de Plan de Formación (83\%) y, además, casi todos ellos basados en diagnósticos de necesidades formativas. La existencia de planes de formación es más frecuente entre las grandes empresas ( $88 \%$ de empresas a partir de 50 empleados frente a un $80 \%$ de las menores a dicho tamaño). Asimismo, la presencia de planes de formación, así como su realización en base a diagnósticos de formación, es ligeramente superior entre las empresas de metalmecánica que en el conjunto del resto de sectores. Existe una relación estadísticamente significativa entre el tamaño y la realización de planes de formación basados en un diagnóstico de necesidades de formación $(\div 2$ sig. $=0,006$ ). Son las empresas más grandes las que en mayor medida realizan sus planes en base a un diagnóstico de necesidades de formación, dado su mayor nivel de formalización en la gestión.

La orientación a la innovación y la existencia de planes de formación en las empresas son dos realidades que aparecen de forma mayoritaria en las empresas analizadas y que están, hasta cierto punto, relacionadas: pocas empresas que se consideran innovadoras carecen de un plan de formación (12\%) y la mayoria de las que no tienen plan de formación (53\%) no se consideran innovadoras. Este hecho apunta hacia la existencia de una relación entre innovación y formación continua: las empresas innovadoras sienten la necesidad de actualizar los recursos y capacidades de sus trabajadores. Entre las empresas innovadoras hay más planes de formación y más planes basados en diagnósticos (esta diferencia es estadísticamente significativa).

TABLA 4. EXISTENCIA DE PLANES DE FORMACIÓN SEGÚN SI LA EMPRESA ES INNOVADORA O NO

\begin{tabular}{|c|c|c|c|c|c|c|c|}
\hline & & \multicolumn{6}{|c|}{ ¿Considera que su empresa es innovadora? } \\
\hline & & \multicolumn{2}{|c|}{ sí } & \multicolumn{2}{|c|}{ NO } & \multicolumn{2}{|c|}{ Total } \\
\hline & & $\mathrm{N}$ & $\%$ & $\mathrm{~N}$ & $\%$ & $\mathrm{~N}$ & $\%$ \\
\hline \multirow{3}{*}{$\begin{array}{l}\text { PLAN DE } \\
\text { FORMACIÓN }\end{array}$} & Basado en DNF & 162 & 75,0 & 76 & 67,3 & 238 & 72,3 \\
\hline & No basado en DNF & 28 & 13,0 & 8 & 7,1 & 36 & 10,9 \\
\hline & No plan de Formación & 26 & 12,0 & 29 & 25,7 & 55 & 16,7 \\
\hline \multicolumn{2}{|l|}{ TOTAL } & 216 & 100,0 & 113 & 100,0 & 329 & 100,0 \\
\hline
\end{tabular}

DNF: Diagnóstico de Necesidades de Formación.

\subsection{Participación de los empleados en actividades de formación}

Las caracteristicas que tratan de medir la implicación de las empresas en las actividades de formación muestran, sobre todo, una gran variabilidad. Seleccionamos como característica descriptiva básica el porcentaje de empleados que reciben formación. El porcentaje de empleados implicados en actividades de formación dentro de las pymes de nuestra muestra es considerablemente elevado, resultando un promedio del $42 \%$. Un 55\% de las empresas declaran que un cuarto o más de su personal ha estado implicado en actividades de formación en el último año. Aunque la situación más frecuente (43\%) es la de las empresas en las que no pasa de un cuarto la plantilla que accede a formación, hay también otro grupo de 31\% para las que más de la mitad de los empleados recibe formación.

El tamaño de la organización incide en la formación. A partir de 75 empleados aumenta de manera estadísticamente significativa $(\chi 2$ sig. $=0,007)$ la cantidad de empresas que tienden a porcentajes medios y altos de la plantilla participando en actividades de formación.

ARBOR Vol. 188753 enero-febrero [2012] 153-170 ISSN: 0210-1963 
TABLA 5. PORCENTAJE DE EMPLEADOS IMPLICADOS EN ACTIVIDADES DE FORMACIÓN

\begin{tabular}{|l|l|c|c|c|c|}
\hline \multicolumn{2}{|c|}{} & \multicolumn{2}{|c|}{ Hasta 75 empleados } & \multicolumn{2}{c|}{75 y más empleados } \\
\cline { 2 - 6 } & Menos del 25\% & N & $\%$ & N & 25 \\
\hline \multirow{3}{*}{$\%$ empleados en formación } & De 25 a 50\% & 115 & 48,7 & 28 & 33,3 \\
\cline { 2 - 7 } & Más del 50\% & 49 & 20,8 & 31 & 36,9 \\
\cline { 2 - 7 } & Total & 72 & 30,5 & 84 & 100,0 \\
\hline
\end{tabular}

También es estadísticamente significativa la relación con las variables "ser una empresa innovadora" y "disponer de un plan de formación" que, como se ha mencionado antes, también están relacionadas entre sí. En estas empresas (innovadoras y/o con planes de formación) la media de participación de trabajadores en actividades de formación es significativamente superior.
Se puede apreciar que, entre las empresas que se consideran innovadoras, la tasa promedio de empleados en formación es del $44,5 \%$, frente al $36 \%$ de las no innovadoras. Esta diferencia se sustenta fundamentalmente en el hecho de que, entre las no innovadoras, la mayoria tiene menos de un cuarto de sus plantillas implicadas en estas actividades mientras que, entre las innovadoras (que son

TABLA 6. PORCENTAJE MEDIO DE EMPLEADOS IMPLICADOS EN ACTIVIDADES DE FORMACIÓN SEGÚN SI LA EMPRESA ES O NO INNOVADORA Y/O DISPONE DE UN PLAN DE FORMACIÓN

\begin{tabular}{|l|c|c|c|}
\hline \multicolumn{2}{|c|}{} & N & Porcentaje medio empleados \\
\hline \multirow{3}{*}{ Empresa innovadora } & Si & 210 & 44,5 \\
\cline { 2 - 4 } & No & 110 & 36,2 \\
\hline \multirow{2}{*}{ Empresa con plan de formación } & Si & 265 & 45,7 \\
\cline { 2 - 4 } & No & 55 & 22,3 \\
\hline TOTAL & & 320 & 41,7 \\
\hline
\end{tabular}

TABLA 7. PORCENTAJES DE EMPLEADOS IMPLICADOS EN ACTIVIDADES DE FORMACIÓN SEGÚN SI LA EMPRESA ES O NO INNOVADORA

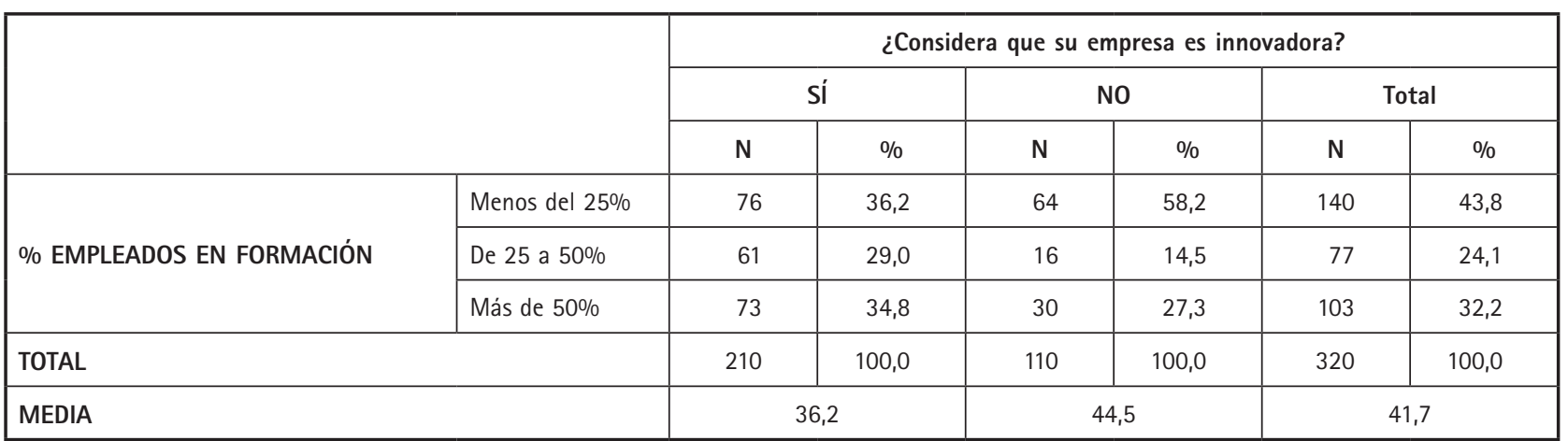


mayoria), hay mucha más variedad de situaciones, pero significativamente más empresas con porcentajes medios y altos de participación en formación.

El sector de actividad no incide en el alcance de las actividades de formación, siendo las medias y distribuciones de participación similares en el sector Metalmecánica y en el resto de empresas.

\section{Relaciones entre pymes y CEnTROS de Formación Profesional}

\subsection{Frecuencia de las relaciones}

Del total de empresas encuestadas, el $82 \%$ afirma que tiene 0 ha tenido alguna relación con centros de FP. Realmente no hay un perfil muy concreto para el pequeño grupo de empresas que dicen no tener esta relación, porque el tipo de motivo más mencionado, con mucha diferencia, es que de alguna manera no se ha planteado la necesidad (67\% de los que no tienen relaciones). Sin embargo, la mayoría son empresas pequeñas (78\% de ellas tienen hasta 50 empleados), empresas que tienen pocos trabajadores participando en actividades de formación (60\% con menos de un cuarto de la plantilla implicada) $y$, sobre todo, que no pertenecen al sector de metalmecánicas (63\% en variedad de sectores). También es relevante destacar de antemano que no se trata de empresas que valoren menos la importancia de los trabajadores con perfil FP para la innovación (cuestión que se discutirá en el apartado sexto).

Aún dentro de lo que es una tendencia absolutamente mayoritaria, las relaciones con centros de FP son significativamente más frecuentes $(\chi 2$ sig $<0,05)$ entre las empresas de mayor tamaño (a partir de 50 empleados el porcentaje asciende al 89,8\%), entre las empresas de metalmecánica (89,5\%), entre las que se consideran innovadoras $(85,6 \%)$, y también entre las que tienen planes de formación $(84,7 \%)$.

TABLA 8. EXISTENCIA DE RELACIONES CON CENTROS DE FP SEGÚN TAMAÑO, SECTOR, SI LA EMPRESA ES INNOVADORA O SI DISPONE DE UN PLAN DE FORMACIÓN

\begin{tabular}{|c|c|c|c|c|c|c|c|}
\hline & \multicolumn{6}{|c|}{$\begin{array}{l}\text { ¿Tiene o ha tenido su empresa alguna experiencia } \\
\text { de relación con algún centro de Formación Profesional (FP)? }\end{array}$} \\
\hline & & \multicolumn{2}{|c|}{ sí } & \multicolumn{2}{|c|}{ NO } & \multicolumn{2}{|c|}{ Total } \\
\hline & & $\mathrm{N}$ & $\%$ & $\mathrm{~N}$ & $\%$ & $\mathrm{~N}$ & $\%$ \\
\hline \multirow{2}{*}{ TAMAÑO } & HASTA 50 & 154 & 76,6 & 47 & 23,4 & 201 & 100,0 \\
\hline & MÁS DE 50 & 115 & 89,8 & 13 & 10,2 & 128 & 100,0 \\
\hline \multirow{2}{*}{ SECTOR } & Empresas de metalmecánica & 188 & 89,5 & 22 & 10,5 & 210 & 100,0 \\
\hline & Resto de empresas & 99 & 71,2 & 38 & 31,9 & 119 & 100,0 \\
\hline \multirow{2}{*}{ Empresa innovadora } & sí & 185 & 85,6 & 31 & 14,4 & 216 & 100,0 \\
\hline & NO & 84 & 74,3 & 29 & 25,7 & 113 & 100,0 \\
\hline \multirow{2}{*}{$\begin{array}{l}\text { Empresa con un Plan } \\
\text { de Formación }\end{array}$} & sí & 232 & 84,7 & 42 & 15,3 & 274 & 100,0 \\
\hline & NO & 37 & 67,3 & 18 & 32,7 & 55 & 100,0 \\
\hline \multicolumn{2}{|l|}{ TOTAL } & 269 & 81,8 & 60 & 18,2 & 329 & 100,0 \\
\hline
\end{tabular}


Otro aspecto importante sobre las relaciones con los centros de FP es que son geográficamente cercanas: el 69\% de las empresas se relacionan principalmente con centros de FP radicados en la misma comarca y hasta en un $89,5 \%$ en su propia provincia. Lógicamente, la distribución tiene que ver directamente con la oferta de los centros en cada territorio y su especialización sectorial en relación con las necesidades de las empresas.

\subsection{Tipos de relaciones}

Respecto a las formas concretas de relación entre empresas y centros FP, se ha trabajado sobre una clasificación muy detallada de tipos de relación (10 tipos) que han resultado de muy distinta importancia en las relaciones detectadas.

El caso más generalizado es el de recibir alumnado en prácticas (87\% de las empresas que tienen alguna relación con centros FP), siendo también mayoritariamente empleado el recurso a la contratación directa de trabajadores de sus Bolsas de Trabajo (58\%). También tienen una incidencia importante las relaciones para la obtención de formación continua, tanto del Catálogo Modular (41\%), como bajo demanda (24\%) así como, en menor medida (16\%), la utilización de las infraestructuras de los centros de FP para actividades de contenido técnico/tecnológico. El resto de las formas de relación, focalizadas en algunos centros de FP, son mucho menos frecuentes dentro de la muestra de pymes de las comunidades autónomas vasca y navarra.

Con relación al nivel de satisfacción de las empresas con estos tipos de experiencias (valoración escala 0-10), se puede detectar unos niveles muy positivos, especialmente entre las experiencias más comunes de cooperación. Dadas las diferencias en el volumen de empresas implicadas en cada formato de relación, la valoración de las experiencias menos comunes debe tratarse con cautela.

En este documento nos centraremos en las actividades de formación continua y en el papel, a nuestro entender importante, que desempeña la cualificación de los trabajadores con perfil de FP en los procesos de innovación.

TABLA 9. TIPOS DE RELACIONES DETECTADAS ENTRE EMPRESAS ENCUESTADAS-CENTROS DE FP Y VALORACIÓN DE LAS MISMAS POR PARTE DE LAS PYMES

\begin{tabular}{|c|c|c|c|c|c|}
\hline & \multicolumn{2}{|c|}{ Relación } & \multicolumn{3}{|c|}{ Satisfacción (0-10) } \\
\hline & sí & $\%$ & $\mathrm{~N}$ & Media & D.T. \\
\hline Recibimos alumnado en prácticas & 233 & 87 & 232 & 7,66 & 1,4 \\
\hline Contratamos trabajadores/as a través de sus Bolsas de trabajo & 156 & 58 & 155 & 7,55 & 1,4 \\
\hline $\begin{array}{l}\text { Nuestros trabajadores/as acuden al centro para recibir cursos de } \\
\text { Formación Continua del Catálogo Modular }\end{array}$ & 111 & 41 & 107 & 7,41 & 1,1 \\
\hline Hemos realizado con el centro Formación Continua "a la carta" & 64 & 24 & 62 & 7,61 & 1,3 \\
\hline $\begin{array}{l}\text { Utilizamos sus instalaciones/maquinaria para efectuar pruebas, } \\
\text { ensayos, etc. }\end{array}$ & 44 & 16 & 43 & 7,42 & 1,2 \\
\hline Estamos en el Patronato del Centro/Fundación & 21 & 7,8 & 17 & 7,18 & 1,1 \\
\hline $\begin{array}{l}\text { Colaboran con nosotros como asesores/consultores en programas } \\
\text { de mejora de la gestión/mejoras organizativas }\end{array}$ & 20 & 7,4 & 19 & 7,16 & 0,9 \\
\hline $\begin{array}{l}\text { Colaboran con nosotros en proyectos (formales) de innovación } \\
\text { técnica }\end{array}$ & 15 & 5,6 & 15 & 6,67 & 1,4 \\
\hline Estamos en la Asociación de Antiguos Alumnos & 12 & 4,5 & 7 & 7 & 1,4 \\
\hline Hemos cedido/financiado maquinaria al centro & 11 & 4,1 & 10 & 6,8 & 1,6 \\
\hline
\end{tabular}




\subsection{La importancia de la formación continua como forma de relación centro de FP-empresa}

\section{Formación continua del Catálogo Modular}

Los centros de FP aparecen como proveedores de considerable importancia en servicios de formación continua para las empresas con las que se relacionan: el $41 \%$ de las empresas envía a sus trabajadores a recibir formación en los centros de FP. Además, el 24\% de las pymes industriales ha desarrollado formación continua bajo demanda con centros de FP. Si bien la combinación de ambos formatos no es muy frecuente $(20,0 \%)$ atendiendo al conjunto de la muestra de empresas que colabora con estos centros (269 pymes), casi la mitad de las empresas que tienen relaciones con centros de $F P$, un 46,0\%, más concretamente, ha recurrido a sus servicios de formación para trabajadores de una u otra forma.

Respecto a la formación continua que las empresas obtienen de los centros a través de cursos del Catálogo Modular, se detecta que los trabajadores de empresas de metalmecánica reciben formación continua en centros de FP en mucha mayor medida (hasta el $48 \%$ de las empresas) que los de otros sectores (26\%), es decir, se da una relación significativa entre el sector y la realización de cursos de formación continua del Catálogo Modular ( $\chi 2$ sig. $=0,001)$. Este hecho pone de manifiesto la especialización de los centros de FP en las familias profesionales más relacionadas con la industria regional. Esta misma relación se plantea también con las empresas innovadoras y con aquellas que disponen de planes de formación.

TABLA 10. REALIZACIÓN DE CURSOS DE FORMACIÓN CONTINUA DEL CATÁLOGO MODULAR EN CENTROS DE FP SEGÚN TAMAÑO, SECTOR, SI LA EMPRESA ES INNOVADORA O DISPONE DE UN PLAN DE FORMACIÓN

\begin{tabular}{|c|c|c|c|c|c|c|c|}
\hline & & \multicolumn{6}{|c|}{$\begin{array}{c}\text { Nuestros trabajadores/as acuden al centro para recibir cursos de Formación } \\
\text { Continua del Catálogo Modular }\end{array}$} \\
\hline & & \multicolumn{2}{|c|}{ sí } & \multicolumn{2}{|c|}{ NO } & \multicolumn{2}{|c|}{ Total } \\
\hline & & $\mathrm{N}$ & $\%$ & $\mathrm{~N}$ & $\%$ & $\mathrm{~N}$ & $\%$ \\
\hline \multirow{2}{*}{ TAMAÑO } & HASTA 50 & 64 & 41,6 & 90 & 58,4 & 154 & 100,0 \\
\hline & MÁS DE 50 & 47 & 40,9 & 68 & 59,1 & 115 & 100,0 \\
\hline \multirow{2}{*}{ SECTOR } & Empresas de metalmecánica & 90 & 47,9 & 98 & 52,1 & 188 & 100,0 \\
\hline & Resto de empresas & 21 & 25,9 & 60 & 74,1 & 81 & 100,0 \\
\hline \multirow{2}{*}{ Empresa innovadora } & sí & 80 & 43,2 & 105 & 56,8 & 185 & 100,0 \\
\hline & NO & 31 & 36,9 & 53 & 63,1 & 84 & 100,0 \\
\hline \multirow{2}{*}{$\begin{array}{l}\text { Empresa con un Plan } \\
\text { de Formación }\end{array}$} & sí & 99 & 42,7 & 133 & 57,3 & 232 & 100,0 \\
\hline & NO & 12 & 32,4 & 25 & 67,6 & 37 & 100,0 \\
\hline \multicolumn{2}{|l|}{ TOTAL } & 111 & 41,3 & 158 & 58,7 & 269 & 100,0 \\
\hline
\end{tabular}

Los cursos de formación que imparten los centros FP para los trabajadores de pymes son muy bien valorados por las empresas: cuando se ha detectado este tipo de relación, las valoraciones resultantes nunca son malas, de manera que, como imagen global, tenemos un notable. La media de valoración de esta relación es de 7,41 puntos en la escala
0-10 y con muy poca dispersión relativa. El consenso es tan amplio en este sentido que no hay diferencias significativas en la valoración según las diversas variables independientes consideradas, excepto para la variable "disponer de un plan de formación": las empresas que disponen de él valoran de manera significativamente mejor la relación 
con los centros de FP a través de los cursos del catálogo Modular que reciben sus trabajadores.

TABLA 11. VALORACIÓN DE LOS DE CURSOS DE FORMACIÓN CONTINUA DEL CATÁLOGO MODULAR EN CENTROS DE FP ENTRE LAS EMPRESAS ENCUESTADAS

\begin{tabular}{|l|c|c|}
\hline & N & $\%$ \\
\hline $0-2$ & 0 & 0 \\
\hline $3-4$ & 0 & 0 \\
\hline $5-6$ & 18 & 16,2 \\
\hline $7-8$ & 74 & 66,7 \\
\hline $9-10$ & 15 & 13,5 \\
\hline NS/NC & 4 & 3,6 \\
\hline Total & 111 & 100,0 \\
\hline Media & & 7,41 \\
\hline
\end{tabular}

\section{Formación continua a medida}

Por otro lado, como se ha señalado, el contacto entre pymes y centros de FP a través de los servicios de formación continua a medida de las necesidades de las empre- sas (formación bajo demanda) tiene una incidencia nada desdeñable entre todos los distintos formatos de relación considerados. En total, casi un 24\% de las empresas que se relacionan con centros FP ha declarado que recurre a ellos para obtener este servicio. Además, la inmensa mayoría de estas empresas $(80,0 \%)$ también envían a sus trabajadores a cursos reglados de reciclaje.

Dos variables caracteristicas de las empresas influyen significativamente en el recurso a la formación "a la carta" con los centros FP: la implicación en innovación y, sobre todo, el tipo de sector de actividad. Dentro de una incidencia que no es mayoritaria, las pymes que se consideran innovadoras y las de metalmecánica recurren a la formación continua de demanda hasta en un 28-29\%, cuando las que no tienen estas características sólo rondan el 13-15\%. Existe una asociación estadisticamente significativa entre realizar formación continua a la carta y pertenecer al sector de metalmecánica $(\chi 2$ sig. $=0,023)$ y/o ser una empresa innovadora $(\chi 2$ sig. $=0,005)$, dando una idea de la confianza y credibilidad de los centros de FP como agente capaz de satisfacer las necesidades de formación de las pymes de este ámbito industrial. A nivel global, el tamaño no parece influir en la incidencia de este tipo de relaciones entre las pymes y los centros de FP.

TABLA 12. REALIZACIÓN DE CURSOS DE FORMACIÓN "A LA CARTA" CON CENTROS DE FP SEGÚN TAMAÑO, SECTOR, SI LA EMPRESA ES INNOVADORA O DISPONE DE UN PLAN DE FORMACIÓN

\begin{tabular}{|c|c|c|c|c|c|c|c|}
\hline & & \multicolumn{6}{|c|}{ Hemos realizado con el centro Formación Continua a la carta } \\
\hline & & \multicolumn{2}{|c|}{ sí } & \multicolumn{2}{|c|}{ NO } & \multicolumn{2}{|c|}{ Total } \\
\hline & & $\mathrm{N}$ & $\%$ & $\mathrm{~N}$ & $\%$ & $\mathrm{~N}$ & $\%$ \\
\hline \multirow[t]{2}{*}{ TAMAÑO } & HASTA 50 & 36 & 23,4 & 118 & 76,6 & 154 & 100,0 \\
\hline & MÁS DE 50 & 28 & 24,3 & 87 & 75,7 & 115 & 100,0 \\
\hline \multirow[t]{2}{*}{ SECTOR } & Empresas de metalmecánica & 52 & 27,7 & 136 & 72,3 & 188 & 100,0 \\
\hline & Resto de empresas & 12 & 14,8 & 69 & 85,2 & 81 & 100,0 \\
\hline \multirow[t]{2}{*}{ Empresa innovadora } & sí & 53 & 28,6 & 132 & 71,4 & 185 & 100,0 \\
\hline & NO & 11 & 13,1 & 73 & 86,9 & 84 & 100,0 \\
\hline \multirow{2}{*}{$\begin{array}{l}\text { Empresa con un Plan } \\
\text { de Formación }\end{array}$} & sí & 59 & 25,4 & 173 & 74,6 & 232 & 100,0 \\
\hline & NO & 5 & 13,5 & 32 & 86,5 & 37 & 100,0 \\
\hline \multicolumn{2}{|l|}{ TOTAL } & 64 & 23,8 & 205 & 76,2 & 269 & 100,0 \\
\hline
\end{tabular}


En este caso de la formación continua bajo demanda o a la carta, y aun tratándose de una forma de relación y servicio menos frecuente, la valoración que realizan las empresas es muy buena, obteniéndose un 7,6 de valoración media. La valoración es significativamente más positiva por parte de las empresas innovadoras frente a las que no lo son.

El recurso a la formación continua a la carta entra dentro de los formatos más intensos de relaciones con los centros FP, ya que el $82,8 \%$ de las empresas que realizan este tipo de formación continua tiene ya otros tres o más tipos de relaciones diferentes con los centros (incluyendo recibir prácticas, contratar egresados de las Bolsas de Trabajo, etc.). Esto confirma la importancia de la formación continua como forma de relación avanzada entre centros de FP y empresas.

TABLA 13. VALORACIÓN DE LOS CURSOS DE FORMACIÓN CONTINUA "A LA CARTA" DE LOS CENTROS DE FP ENTRE LAS EMPRESAS ENCUESTADAS

\begin{tabular}{|l|c|c|}
\hline \multirow{2}{*}{} & \multicolumn{2}{|c|}{ Muestra total } \\
\cline { 2 - 3 } & $\mathbf{N}$ & $\%$ \\
\hline $0-2$ & 1 & 1,6 \\
\hline $3-4$ & 0 & 0 \\
\hline $5-6$ & 6 & 9,4 \\
\hline $7-8$ & 44 & 68,8 \\
\hline $9-10$ & 11 & 17,2 \\
\hline NS/NC & 2 & 3,1 \\
\hline Total & 64 & 100,0 \\
\hline Media & \multicolumn{2}{|c|}{7,61} \\
\hline
\end{tabular}

Impacto de la formación continua en las competencias de los trabajadores y en la capacidad de innovación de la empresa

Este trabajo nos ha permitido, también, conocer cuál es la valoración que efectúan las empresas encuestadas sobre el impacto de la formación continua impartida en los centros de FP en la motivación, valor y productividad de los trabajadores. Asimismo, nos ofrece una imagen de la percepción que las empresas tienen sobre la aportación global de los trabajadores reciclados en estos centros a los procesos de innovación de las empresas.

Para ello, hemos utilizado una medición de grados de acuerdo (escala 0-10) con una serie de cuatro dimensiones de posibles consecuencias o impactos positivos de la formación continua sobre la capacitación de los trabajadores y la innovación de las empresas. Según los datos obtenidos, se puede concluir que, a nivel global, hay un acuerdo claro y mayoritario en que la formación continua realizada por los centros FP tiene efectos positivos en tres aspectos concretos: facilita una mayor motivación de los trabajadores (60\% claramente de acuerdo con puntuaciones 7-10), mejora la productividad de los trabajadores (54\%) y ha permitido a las empresas tener un personal más valioso que el de la competencia (51\%).

Preguntadas las empresas si gracias a la formación continua son más innovadoras, las respuestas están más divididas. No obstante, un porcentaje nada desdeñable de empresas $(41,2 \%)$ considera que existe una relación directa y positiva entre formación continua e innovación.

TABLA 14. VALORACIÓN DEL IMPACTO DE LA FORMACIÓN CONTINUA QUE IMPARTEN LOS CENTROS DE FP EN LAS COMPETENCIAS DE LOS TRABAJADORES Y EN LA CAPACIDAD DE INNOVACIÓN DE LA EMPRESA

\begin{tabular}{|c|c|c|c|c|c|c|c|c|}
\hline & \multicolumn{2}{|c|}{$\begin{array}{l}\text { Mayor motivación } \\
\text { trabajadores }\end{array}$} & \multicolumn{2}{|c|}{$\begin{array}{l}\text { Personal más valioso que } \\
\text { competencia }\end{array}$} & \multicolumn{2}{|c|}{$\begin{array}{l}\text { Mayor productividad } \\
\text { trabajadores }\end{array}$} & \multicolumn{2}{|c|}{$\begin{array}{c}\text { Mayor grado innovación } \\
\text { en empresa }\end{array}$} \\
\hline & $\mathrm{N}$ & $\%$ & $\mathrm{~N}$ & $\%$ & $\mathrm{~N}$ & $\%$ & $\mathrm{~N}$ & $\%$ \\
\hline $0-2$ & 4 & 3,2 & 6 & 4,8 & 6 & 4,8 & 17 & 13,7 \\
\hline $3-4$ & 7 & 5,6 & 7 & 5,6 & 11 & 8,9 & 16 & 12,9 \\
\hline $5-6$ & 36 & 29,0 & 36 & 29,0 & 39 & 31,5 & 38 & 30,6 \\
\hline $7-8$ & 62 & 50,0 & 51 & 41,1 & 58 & 46,8 & 42 & 33,9 \\
\hline $9-10$ & 12 & 9,7 & 13 & 10,5 & 9 & 7,3 & 9 & 7,3 \\
\hline $\mathrm{NS} / \mathrm{NC}$ & 3 & 2,4 & 11 & 8,9 & 1 & 8 & 2 & 1,6 \\
\hline Total & 124 & 100,0 & 124 & 100,0 & 124 & 100,0 & 124 & 100,0 \\
\hline Media & \multicolumn{2}{|c|}{6,62} & \multicolumn{2}{|c|}{6,52} & \multicolumn{2}{|c|}{6,33} & \multicolumn{2}{|c|}{5,59} \\
\hline
\end{tabular}


Es interesante señalar que las empresas que han realizado formación continua a la carta valoran de manera significativamente más positiva el impacto de la formación recibida de los centros de FP en los procesos de innovación en su empresa (valoración media de 6 puntos frente a 5,1 de media para las que no tienen esta experiencia concreta).

Por otro lado, las empresas con un plan de formación valoran de manera significativamente más positiva el impacto de la formación en una mayor motivación de los trabajadores, en una mayor productividad y en un mayor grado de innovación de la empresa.

\section{IMPORTANCIA DE LOS TRABAJADORES DE FP EN LOS PROCESOS DE INNOVACIÓN}

Además de estudiar las distintas formas de relación entre centros de FP y empresas, nos ha parecido interesante considerar, siquiera de un modo exploratorio, la percepción de las empresas sobre la aportación de los trabajadores con perfil de FP a los procesos de innovación.

Las empresas han valorado la importancia de estos trabajadores en las actividades de innovación mediante una escala 0-10 y en cuatro aspectos concretos; a saber: el desarrollo de nuevos productos, el desarrollo de nuevos procesos, la implantación de nuevas tecnologías y la implantación de nuevos sistemas organizativos.
En general, las valoraciones positivas (5-10) sobre la importancia de los trabajadores con perfil de FP en las actividades de innovación superan a las negativas para todos los aspectos, es decir, que, para la mayoría de pymes industriales, los trabajadores con perfil FP son realmente importantes en los procesos de innovación que acometen. El tipo de innovación para el que comparativamente se concede mayor importancia a la participación de los trabajadores es el desarrollo de nuevos procesos (media de 5,85).

En cualquier caso, es de destacar que no menos de un 34\% (y hasta un 43\%) de las empresas encuestadas afirman que los trabajadores con perfil de FP tienen una considerable importancia para algún aspecto de innovación (valoraciones 7-10).

No se aprecian diferencias significativas de valoración según tamaño $y$, entre las empresas de metalmecánica y el resto. Sin embargo, las empresas que se consideran innovadoras y/o disponen de un Plan de Formación parecen otorgar una mayor importancia a los trabajadores en los procesos de innovación.

Por otro lado, se han considerado los factores que dificultan la participación de los trabajadores en los procesos de innovación. Hay dos cuestiones que aparecen para las pymes industriales como comparativamente más problemáticas: la falta de motivación y la falta de capacitación de los trabajadores (valoraciones medias de relevancia de 5,38 y 5,15 respectivamente). Entre las dificultades valo-

TABLA 15. VALORACIÓN DE LA IMPORTANCIA DE LOS TRABAJADORES DE FP EN LOS PROCESOS DE INNOVACIÓN

\begin{tabular}{|c|c|c|c|c|c|c|c|c|}
\hline & \multicolumn{2}{|c|}{$\begin{array}{c}\text { Desarrollo } \\
\text { de nuevos productos }\end{array}$} & \multicolumn{2}{|c|}{$\begin{array}{c}\text { Desarrollo } \\
\text { de nuevos procesos }\end{array}$} & \multicolumn{2}{|c|}{$\begin{array}{c}\text { Implantación } \\
\text { de nuevas tecnologías }\end{array}$} & \multicolumn{2}{|c|}{$\begin{array}{l}\text { Implantación nuevos } \\
\text { métodos organizativos }\end{array}$} \\
\hline & $\mathrm{N}$ & $\%$ & $\mathrm{~N}$ & $\%$ & $\mathrm{~N}$ & $\%$ & $\mathrm{~N}$ & $\%$ \\
\hline $0-2$ & 58 & 17,6 & 37 & 11,2 & 54 & 16,4 & 55 & 16,7 \\
\hline $3-4$ & 35 & 10,6 & 26 & 7,9 & 34 & 10,3 & 34 & 10,3 \\
\hline $5-6$ & 67 & 20,4 & 88 & 26,7 & 79 & 24,0 & 91 & 27,7 \\
\hline $7-8$ & 109 & 33,1 & 116 & 35,3 & 104 & 31,6 & 92 & 28,0 \\
\hline $9-10$ & 21 & 6,4 & 27 & 8,2 & 21 & 6,4 & 20 & 6,1 \\
\hline NS & 39 & 11,9 & 35 & 10,6 & 37 & 11,2 & 37 & 11,2 \\
\hline Total & 329 & 100,0 & 329 & 100,0 & 329 & 100,0 & 329 & 100,0 \\
\hline Media & \multicolumn{2}{|c|}{5,29} & \multicolumn{2}{|c|}{5,85} & \multicolumn{2}{|c|}{5,34} & \multicolumn{2}{|c|}{5,24} \\
\hline
\end{tabular}


TABLA 16. VALORACIÓN DE LOS FACTORES OUE DIFICULTAN (0-10) UNA MAYOR PARTICIPACIÓN DE LOS TRABAJADORES CON ESTUDIOS DE FP EN LOS PROCESOS DE INNOVACIÓN

\begin{tabular}{|c|c|c|c|c|c|c|c|c|c|c|c|c|}
\hline & \multicolumn{2}{|c|}{$\begin{array}{c}\text { Falta capacitación } \\
\text { trabajadores }\end{array}$} & \multicolumn{2}{|c|}{$\begin{array}{l}\text { Falta motivación } \\
\text { trabajadores }\end{array}$} & \multicolumn{2}{|c|}{$\begin{array}{l}\text { Falta incentivos } \\
\text { para trabajadores }\end{array}$} & \multicolumn{2}{|c|}{$\begin{array}{c}\text { Barreras } \\
\text { organizativas } \\
\text { empresa }\end{array}$} & \multicolumn{2}{|c|}{$\begin{array}{l}\text { Falta de confianza } \\
\text { en trabajadores }\end{array}$} & \multicolumn{2}{|c|}{$\begin{array}{l}\text { Ausencia cultura } \\
\text { cooperación }\end{array}$} \\
\hline & $\mathrm{N}$ & $\%$ & $\mathrm{~N}$ & $\%$ & $\mathrm{~N}$ & $\%$ & $\mathrm{~N}$ & $\%$ & $\mathrm{~N}$ & $\%$ & $\mathrm{~N}$ & $\%$ \\
\hline $0-2$ & 63 & 19,1 & 57 & 17,3 & 63 & 19,1 & 84 & 25,5 & 93 & 28,3 & 66 & 20,1 \\
\hline $3-4$ & 42 & 12,8 & 50 & 15,2 & 48 & 14,6 & 50 & 15,2 & 68 & 20,7 & 50 & 15,2 \\
\hline $5-6$ & 88 & 26,7 & 66 & 20,1 & 90 & 27,4 & 82 & 24,9 & 77 & 23,4 & 84 & 25,5 \\
\hline 7-8 & 69 & 21,0 & 86 & 26,1 & 83 & 25,2 & 61 & 18,5 & 48 & 14,6 & 74 & 22,5 \\
\hline 9-10 & 33 & 10,0 & 39 & 11,9 & 12 & 3,6 & 16 & 4,9 & 12 & 3,6 & 24 & 7,3 \\
\hline NS & 34 & 10,3 & 31 & 9,4 & 33 & 10,0 & 36 & 10,9 & 31 & 9,4 & 31 & 9,4 \\
\hline Total & 329 & 100,0 & 329 & 100,0 & 329 & 100,0 & 329 & 100,0 & 329 & 100,0 & 329 & 100,0 \\
\hline Media & \multicolumn{2}{|c|}{5,15} & \multicolumn{2}{|c|}{5,38} & \multicolumn{2}{|c|}{4,81} & \multicolumn{2}{|c|}{4,37} & \multicolumn{2}{|c|}{3,98} & \multicolumn{2}{|c|}{4,86} \\
\hline
\end{tabular}

radas, se trata de los dos aspectos que pueden atribuirse más directamente a los propios trabajadores. Los obstáculos que aparecen como menos problemáticos para los encuestados (directivos de las pymes), en general, son las rigideces organizativas $y$, especialmente, la falta de confianza de la dirección.

El tamaño es relevante en el análisis de estas dimensiones: las empresas más pequeñas (por debajo de 50 trabajadores) ven todos los factores como más problemáticos que las empresas más grandes, existiendo una relación significativa al respecto. Para el resto de variables potencialmente explicativas (innovación, planes de formación o sector) no se han detectado diferencias significativas de resultados.

\section{Conclusiones}

Por medio del presente trabajo hemos pretendido aumentar el conocimiento existente sobre la percepción que las empresas tienen de los centros de FP y sobre la relación que se produce entre ambos agentes. Se ha prestado especial atención a las actividades de formación continua -papel emergente dentro de los desarrollados por los centros de FP en España- y su posible relación con los procesos de innovación en las empresas (pymes industriales).
Una de las aportaciones de este trabajo es la de constatar que, en un contexto de insuficiente conexión entre el sistema educativo y el sistema productivo en España, en las comunidades analizadas la relación de colaboración entre pymes y centros de FP es fluida y fructífera. El $82 \%$ de las empresas encuestadas ha tenido alguna relación de colaboración con los centros de FP.

Es necesario resaltar el alto nivel de incidencia de las formas de relación más tradicionales, como la FCT y el recurso a las Bolsas de Trabajo de los centros, conducentes ambas a dotar a las empresas de un capital humano más adaptado a sus necesidades técnico-productivas. Un $87 \%$ de las empresas que mantienen relaciones con los centros de FP han recibido alumnado en prácticas. La valoración que las empresas hacen de estas relaciones es muy satisfactoria. Dentro también de las funciones clásicas de la FP, es remarcable que la mayoría de las empresas que colaboran con los centros de FP recurren a sus bolsas de trabajo, al igual que en el caso anterior, con elevados índices de satisfacción con el servicio recibido.

La formación continua, aunque no es una forma de relación tan extendida, presenta una destacada incidencia: el 46\% de las empresas que han tenido relación con un centro de FP han participado en estas actividades, bien a través de los cursos del Catálogo Modular (42\%) o en cursos bajo

ARBOR Vol. 188753 enero-febrero [2012] 153-170 ISSN: 0210-1963 
demanda (24\%). La valoración que las empresas realizan de estas formas de relación es también muy positiva.

Los resultados obtenidos en la encuesta permiten afirmar que la formación continua se ha consolidado como una relación de colaboración privilegiada y central entre los centros de FP y las empresas. Las empresas que realizan más formación continua muestran un mayor acuerdo sobre los efectos de la formación en la motivación, la productividad y la aportación de los trabajadores. El nivel de acuerdo sobre los efectos directos en la innovación es menor, pero no desdeñable. Es interesante resaltar que las empresas que han realizado formación continua bajo demanda $y_{\text {, }}$ por tanto, adecuada especificamente a sus necesidades, valoran más los efectos en la innovación. Este tipo de formación es significativamente mejor valorado por las pymes innovadoras, y es además un indicador de la intensidad de la relación centro de FP/empresa. El 83\% de las empresas que realizan este tipo de formación acumula otros tres 0 más tipos de relación con los centros. Así las cosas, a medida que aumenta el conocimiento mutuo y la confianza entre empresas y centros de FP, se van intensificando las relaciones de colaboración, hasta llegar al desarrollo de las funciones menos tradicionales de la FP, a saber: ser un colaborador en formación continua y, en los casos más avanzados, en servicios para la innovación.

Los resultados obtenidos permiten también constatar la existencia de sistemas locales de producción e innovación en las comunidades autónomas analizadas, tal y como sugiere la literatura internacional: las empresas de metalmecánica, innovadoras y que cuentan con un plan de formación realizan significativamente más formación continua, tanto modular como bajo demanda. Por otro lado, considerando todos los tipos de colaboración pyme-centro de $\mathrm{FP}$, el 69\% de dichas colaboraciones se producen con centros de la comarca en la que se radica la empresa (máximo de $20 \mathrm{~km}$ a la redonda), y el 90\%, con centros de la misma provincia. Este hecho pone de manifiesto la importancia de esta infraestructura de conocimiento en el ámbito regional.

Otras formas de relación centro/empresa no tradicionales, como los proyectos de innovación, que han comenzado a ser apoyadas por algunas administraciones regionales y que son desarrolladas, focalizadamente, por los centros más punteros, presentan en la actualidad una incidencia muy escasa a nivel general.

No obstante, los resultados de este trabajo sugieren que la Formación Profesional, como sistema, también incide en los procesos de innovación a través del capital humano. A este respecto, se ha medido, siquiera tentativamente, la percepción de la contribución que estos trabajadores/as, técnicos cualificados, pueden realizar en los procesos de innovación. La valoración que las empresas hacen de esta aportación no es desdeñable, pero es bastante inferior a realizada respecto a otros aspectos de la formación profesional. Las pymes innovadoras y las que cuentan con un plan de formación valoran más positivamente la aportación que los técnicos de FP pueden realizar en los procesos de innovación, pero siempre en valores que no superan, por término medio, los 6 puntos (sobre 10). En estudios futuros sería conveniente incidir en las potencialidades de una mayor participación y en las barreras que la puedan estar impidiendo. Asimismo, sería de gran interés conocer qué competencias son las que, trabajadas en la Formación Profesional, se transfieren a las empresas facilitando los procesos de innovación en éstas.

En suma, los resultados obtenidos avalan la pertinencia de atender a las relaciones entre el sistema de innovación y el sistema de formación, aspecto que ha sido descuidado en los estudios recientes sobre la innovación.

\section{NOTAS}

1 Este estudio se ha realizado con financiación de la Diputación Foral de Gipuzkoa (Red Guipuzcoana de Ciencia, Tecnología e Innovación), del

Recibido: 8 de enero de 2011 Aceptado: 1 de abril de 2011
Gobierno Vasco (Ayudas a grupos de investigación del sistema universita- rio vasco) y del Ministerio de Ciencia e Innovación (proyecto CSO200806520-C02-01)

2 Tampoco hay que olvidar que el porcentaje de población con un nivel educativo obligatorio o inferior es del 49\%, 19 puntos por encima de la media de la OCDE (30\%). La situación está 
mejorando rápidamente a este respecto (20 puntos en los últimos once años) pero las diferencias con la OCDE, que también ha mejorado su porcentaje en 7 puntos en dicho periodo, siguen siendo muy significativas.

3 Las tasas brutas de escolarización se calculan como la relación entre el total de alumnado de cualquier edad de la enseñanza considerada y la población del grupo de edad teórica de cursar dicha enseñanza. Las edades teóricas utilizadas han sido las siguientes: Ciclos Formativos de Grado Medio, 16-17 años; Ciclos Formativos de Grado Superior, 18-19 años.

4 Para la identificación del marco poblacional y Planificación de la muestra se ha utilizado la base de datos $\mathrm{SABI}$ de Bureau van Dick.

5 Para la realización del trabajo de campo, el equipo de investigación ha enviado a las 1.718 empresas del marco poblacional una carta requiriendo la colaboración en el presente estudio. El trabajo de campo se subcontrató a una empresa especializada, que se encargó de realizar las encuestas telefónicas, que tuvieron lugar entre el 10 y el 24 de junio de 2010.

6 La agrupación corresponde exactamente a los códigos de actividad 27, 28, 29, 31, 34 y 35 (CNAE93rev a 2 dígitos): Metalurgia; Fabricación de productos metálicos; Industria de la construcción de maquinaria y equipo mecánico; Fabricación de maquinaria y material eléctrico; Fabricación de vehículos de motor, remolques y semirremolques; y Fabricación de otro material de transporte.

\section{REFERENCIAS BIBLIOGRÁFICAS}

Arundel, A.; Lorenz, E.; Lundvall, B. A. y Valeyre, A. (2007), "How Europe's economies learn: A comparison of work organization and innovation mode for the EU-15", Industrial and Corporate Change, vol. 16, n. ${ }^{\circ} 6$, pp. 1175-1210.

Asheim, B. T. y Coenen, L. (2005), "Knowledge bases and regional innovation systems: comparing nordic clusters", Research policy, 34 (8): pp. 173-1190.

Bosch, G. y Charest, J. (2008), "Vocational training and the labour market in liberal and coordinated economies", Industrial Relations Journal, 39(5), pp. 428-447.

Berechet, C.; Huerta, E. y San Miguel, F. (2008), Invertir en las personas: Formación y productividad en Navarra, Centro para la Competitividad en $\mathrm{Na-}$ varra, Institución Futuro.

Braczyk, H.; Cooke, P. y Heidenreich, R. (Eds.) (1996), Regional Innovation Systems. The Role of Governances in a Globalized World, London, University College London Press.

CEDEFOP (2009), Competencia internacional de alto nivel en formación profesional: las economías emergentes, CENDEFOP, Hoja Informativa.

CES (2009), Sistema educativo y capital humano, Madrid, Consejo Económico y Social.

Cooke, P. y Morgan. K. (1998), The associational economy: Firms, regions and innovation, Oxford, Oxford University Press.

Curtain, R. (2004), "Innovation and Vocational Education and Training", en Dawe, S. (ed.), Vocational Education and Training and Innovation: Research Readings, Adelaide, SA, Australia, National Centre for Vocational Education Research (NCVER), pp. 42-58.

Doloreux, D. (2003), "Regional innovation systems in the periphery: the case of Beauce in Quebec (Canada)", International Journal of innovation management, 7 (1), pp. 67-94.
Dosi, G.; Freeman, C.; Nelson, R. R.; Silverberg, G. y Soete, L. (Eds.) (1988), Technological Change and Economic Theory, London, Pinter.

Edquist, C. (1997), Systems of Innovation: Technologies, Institutions, and Organizations, London, Pinter.

Freeman, C. (1987), Technology, Policy, and Economic Performance: Lessons from Japan, London, Pinter Publishers.

Gebauer, A.; Woon Nam, C. y Parsche, R. (2005), "Regional technology policy and factors shaping local innovation networks in small German cities", European Planning Studies, 13 (5), pp. 661-683.

Grotz, R. y Braun, B. (1997), "Territorial or transnational networking: spatial aspects of technology oriented cooperation within the German mechanical Engineering Industry", Regional Studies, 31 (6), pp. 545-557.

Guthrie, H. y Dawe, S. (2004), "Overview", en Dawe S (ed.), Vocational Education and Training and Innovation: Research Readings, Adelaide, SA, Australia, NCVER, pp. 10-19.

Homs, 0. (2008), La formación profesional en España: Hacia la sociedad del conocimiento, Barcelona: Fundación "la Caixa".

Huerta, E. (ed.) (2003), Los desafíos de la competitividad: La innovación organizativa y tecnológica en la empresa española, Bilbao, Fundación BBVA.

Isaksen, B. T. y Asheim, A. 2003 "SMEs and the regional dimension of innovation", en Asheim, B. T.; Isaksen, A.; Nauwelaers C. y Tödtling, F. (eds.): Regional innovation policy for small-medium enterprises, Cheltenham, Edwar Elgar, PP. 21-46.

Kaufmann, A. y Todtling, F. (2003), "Innovation pattern of SMEs", en B. Asheim, A. Isaksen, C. Nauwelaers y F. Tödtling (Eds.), Regional innovation policy for small-medium enterprises, Cheltenham, Edwar Elgar, pp. 78-115. 
Lundvall, B. A. (1992a), National Systems of Innovation: Towards a Theory of Innovation and Interactive Learning, London, Pinter.

Lundvall, B. A. (2002), Innovation, Growth and Social Cohesion: The Danish Model, Cheltenham UK, Edward Elgar.

Lundvall, B. A. y Christensen J. L. (2003), Broadening the Analysis of Innovation Systems - Competition, Organisational. Lundvall, B. A.; Rasmussen, P. y Lorenz, E. (2008), "Education in the Learning Economy: A European Perspective", Policy Futures in Education, vol. 6, n. 6, pp. 681-700.

Maskell, P. y Malmberg, A. (1999), "Localised learning and industrial competitiveness", Cambridge Journal of Economics, 23 (2), pp. 167-186.

Moodie, G. (2006), "Vocational education institutions' role in national innovation", Research in Post-compulsory Education, vol. 11, n. ${ }^{\circ}$ 2, pp. 131-140.
Nelson, R. R. (Ed.) (1993), National Innovation Systems: A Comparative Analysis, Oxford, Oxford University Press.

OECD (2009), Education at a Glance, Paris, OCDE.

Rosenfeld, S. (1998), Technical colleges, technology deployment and regional development, Modena, Italy, OECD.

Ranga, L. M.; Miedema, J. y Jorna, R. (2008), "Enhancing the innovative capacity of small firms through triple helix interactions: challenges and opportunities", Technology Analysis and Strategic Management, vol. 20, n. ${ }^{\circ}$, pp. 697-716.

Storper, M. (1997), The regional economy, New York, Guilford Press.

Tether, B.; Mina, A.; Consoli, D. y Gagliardi, D. (2005), A Literature Review on Skills and Innovation. How Does Successful Innovation Impact on the Demand of Skills and How Do Skills Drive Innovation?, Manchester, ESRC
Centre for Research on Innovation and Competition (CRIC), University of Manchester.

Toner, P. (2009), Workforce Skills and Innovation: An Overview of Major Themes in the Literature, Paris, OCDE.

Toner, P.; Marceau, J.; Hall, R.; Considine, G. (2004), "Innovation agents: Vocational education and training skills in the present and future Australian innovation systen", en Dawe, S. (ed.), Vocational Education and Training and Innovation: Research Readings, Adelaide, SA, Australia, NCVER, pp. 84105.

Whittingham, K.; Ferrier, F. y Trood, C. (2004), "Vocational education and training and the commercialisation of Australian research", en Dawe, S. (ed.), Vocational Education and Training and Innovation: Research Readings, Adelaide, SA, Australia, NCVER, pp. 106-117. 